\title{
French Pharmacovigilance Public System and COVID-19 Pandemic
}

\author{
Aurélie Grandvuillemin ${ }^{1}$ (] $\cdot$ Milou-Daniel Drici ${ }^{2}$. Annie Pierre Jonville-Bera ${ }^{3}$. Joelle Micallef ${ }^{4}$. \\ Jean Louis Montastruc ${ }^{5}$ the French Pharmacovigilance Network
}

Accepted: 12 December 2020 / Published online: 28 December 2020

(c) The Author(s), under exclusive licence to Springer Nature Switzerland AG part of Springer Nature 2020

\begin{abstract}
The current COVID-19 pandemic is an exceptional health situation including for drug use. As there was no known effective drug for COVID-19 at the beginning of the pandemic, different candidates were proposed. In this short article, we present the French public pharmacovigilance activities during this health crisis. Although COVID-19 is a confounding factor per se, owing to its potential for multi-organ damage including the heart and kidney, the quality of the transmitted data in adverse drug reaction reports, the timeliness of feedback from clinicians, and the real-time pharmacological and medical analysis by the French network of the regional pharmacovigilance centers made it possible to swiftly identify relevant safety signals. The French National Agency of Medicine was thus able to validate the data and convey their findings very early. This decentralized organization based on medical and pharmacological evaluation of case reports has proven to be efficient and responsive in this unique and challenging healthcare emergency.
\end{abstract}

Aurélie Grandvuillemin

aurelie.grandvuillemin@chu-dijon.fr

1 Centre régional de Pharmacovigilance, Hôpital François Mitterrand, 14 Rue Paul Gaffarel, BP77908, Dijon 21079, France

2 Regional Pharmacovigilance Center of Nice, Department of Clinical Pharmacology, Université Côte d'Azur, Nice, France

3 Regional Pharmacovigilance Center of Tours, Service de Pharmacosurveillance, CHRU, Tours, France

4 Regional Pharmacovigilance Center of Marseille, Service Hospitalo-Universitaire de Pharmacologie Clinique et Pharmacovigilance, Assistance Publique Hôpitaux de Marseille, Aix Marseille Université, Institut des Neurosciences des Systèmes, INSERM 1106, Marseille, France

5 Regional Center of Pharmacovigilance, Pharmacoepidemiology and Drug Information, Service de Pharmacologie Médicale et Clinique, IC 1436 INSERM, Faculty of Medicine, University Hospital, Toulouse, France

\section{Key Points}

Real-time medical and pharmacological evaluation of adverse drug reaction reports is essential to ensure efficiency and responsiveness needed in such a health crisis.

Monitoring of a "known" drug is still crucial as any drug taken out of its usual context may have a different safety profile with new, more frequent, or serious adverse drug reactions.

\section{Introduction}

The current pandemic is an exceptional health situation on many different levels: first, a new virus (SARS-CoV2) has been identified, making it unique from a virological standpoint; second, is it pathologically unique becasuse the related disease known as COVID-19 has emerged with different clinical presentations [1]; and third, it is a pharmacological challenge because there was (and currently there is still) no known effective drug for COVID-19. 


\section{First Weeks of Pandemic: A Pharmacological Challenge}

This new virus was first reported on 31 December, 2019 by the Wuhan Municipal Health Commission, in Hubei Province (China). On 11 February, the World Health Organization (WHO) named the new disease "COVID-19". On 11 March, given the rapid and extensive spread of disease that was responsible for several thousand deaths within a short time period, the WHO classified it as a pandemic [2].

In France, the framework for the clinical management of patients with COVID-19 was provided by reports from the High Council for Public Health, first published on 5 March, 2020 [3]. This report was based on a scientific evaluation using the data available at that time, including the "WHO R\&D Blueprint: informal consultation on prioritization of candidate therapeutic agents for use in novel coronavirus 2019 infection" published on 24 January [4]. It is important to specify that these data were, and still are, progressing and should be analyzed with regard to their date of publication. While clinical trials are ongoing, the health emergency has led to the consideration of candidate agents on the basis of in vitro results or data obtained from other coronavirus diseases. The first candidates, including repurposed approved drugs, were [3, 5]:

- Remdesivir, developed for the treatment of Ebola disease and for which data were available for other coronaviruses (SARS-CoV and MERS-CoV). Specific pharmacodynamic data for SARS-CoV2 have been available as of February 2020.

- Combination lopinavir/ritonavir, used since the early 2000 s in the treatment of human immunodeficiency virus and for which data have been available for other coronaviruses (SARS-CoV and MERS-CoV).

- Chloroquine, and secondarily hydroxychloroquine, for their potential antiviral action (in vitro data available for SARS-CoV and MERS-CoV). In vitro data for SARS-CoV2 have been available since February 2020 for chloroquine and March 2020 for hydroxychloroquine.

Most of these drugs are not "new" because they have already been used in other indications, sometimes for long periods of time: chloroquine has been used in malaria for several decades, hydroxychloroquine in autoimmune diseases, and lopinavir/ritonavir combination therapy in human immunodeficiency virus. Even though these are "known" drugs, it was immediately considered crucial to monitor adverse drug reactions (ADRs) to detect new safety signals related to the administration of these drugs in a new disease characterized by pulmonary and extrapulmonary manifestations [6]. In fact, the pathophysiological context can modify the safety profile of a drug used in another indication. Similarly, its safety profile can be modified after the use of new dosages or drug associations. These new conditions of drug utilization can lead to the identification of new, more frequent, or serious ADRs.

\section{From First ADR Reports to Public Announcements}

On 26 March, 2020, using data submitted by health professionals, the network of 31 French regional pharmacovigilance centers (RPVCs) was able to alert the National Agency for the Safety of Medicines and Health Products (ANSM) about the occurrence of two serious cases of cardiovascular ADRs reported with hydroxychloroquine. A specific monitoring committee bringing together representatives from ANSM and RPVCs and a national pharmacovigilance survey were set up on 27 March. The objective of this investigation, which is ongoing, was to detect safety signals through targeted monitoring of ADRs of drugs used in patients treated for COVID-19. The scope of the survey was not only ADRs for drugs used outside their marketing authorization, but also, more broadly, ADRs for all drugs administered to these patients. In addition, the investigation aimed to detect drugs that could be suspected of promoting the infection or causing a more serious form of the disease than expected. Taking into account the already known safety profile of hydroxychloroquine and the first case reports notified to RPVCs, an immediate focus was placed on cardiac ADRs. Furthermore, given the significant risk of drug misuse in this context, a regional pilot study was extended to the whole country. The study was designed to give community-based pharmacists the opportunity to report misuse without ADRs to RPVCs through a simple online questionnaire (https://www.proje t-mesange.fr/). Misuse of psychoactive drugs was already monitored by the French Addictovigilance Network [7].

Since then, ADRs collected by the French network of RPVCs have been analyzed on a daily basis, and weekly reports are discussed by a specific monitoring committee. Information relating to the vigilance of ongoing clinical trials in France as well as information from the French Addictovigilance Network and the French Poison Control and Toxicovigilance Network was also integrated. The resulting reports are posted online on the French drug agency site to provide complete transparency (www.ansm.sante.fr).

The warning signals concerning cardiac ADRs for hydroxychloroquine, alone and/or in combination with azithromycin (as published later [8]), but also for lopinavir/ritonavir, were identified early [9], resulting in a public announcement from the ANSM on 30 March, 2020 [10]. Over the following days and weeks, this signal was 
reinforced, and very early on, RPVCs concluded to an unfavorable benefit-harm balance for these drugs outside of clinical trial use. Pharmacovigilance data were exchanged with other institutions, including the High Council for Public Health and the European Medicines Agency.

In April and May 2020, international data gradually came to provide additional information for the benefit-harm assessment of hydroxychloroquine. These data led the High Council for Public Health, in its report published on 24 May, to advise against its use in ambulatory or hospitalized patients, regardless of the seriousness [11]. The European Medicines Agency, for its part, communicated on the risks associated with hydroxychloroquine and chloroquine on 23 April, 2020 and the US Food and Drug Administration on 24 April, 2020.

In France, the decree authorizing the use of hydroxychloroquine and lopinavir/ritonavir in COVID-19, excluding clinical trials, was repealed on 26 May, 2020. The US Food and Drug Administration announced a similar decision on 15 June, 2020 [12].

Other safety concerns have been discussed, especially the involvement of non-steroidal anti-inflammatory drugs in a more serious form of COVID-19 than expected [13, 14]. On their website, the French network of RPVCs has provided information for health professionals and the general public on the risks of hydroxychloroquine and chloroquine since 22 March and more specifically for cardiac risk since 27 March (https://www.rfcrpv.fr/). Moreover, RPVCs are participating in the COVID-19 frequently asked questions of the French Society of Pharmacology and Therapeutic website (https:// sfpt-fr.org/covid19). The specific monitoring committee and the survey are still ongoing while the "second wave" is hitting France as well as Europe.

\section{Conclusions}

This exceptional health situation has confirmed the usefulness of reporting ADRs, or suspected ADRs, even for established medicines that we believe we already understand. Our experience with COVID-19 has also served as a reminder that any drug taken out of its usual context can potentially be more harmful than helpful. This health crisis has provided clear proof of the major interest of the current French public pharmacovigilance system, which is based on direct reporting of ADRs by healthcare professionals and patients to the clinical pharmacologists from the RPVCs. Although COVID-19 is a confounding factor per se, with its potential for multi-organ damage that includes the heart, the quality of the transmitted data, the timeliness of feedback from clinicians, and the real-time pharmacological and medical analysis by RPVC professionals made it possible to swiftly identify relevant safety signals. The ANSM was thus able to validate the data and convey their findings very early. The French pharmacovigilance system, based on the network of the RPVCs, has proven to be efficient and responsive in this unique and challenging healthcare emergency. Some pharmacovigilance systems are working on automated signal detection by using tools connected to very large databases. However, for the time being, these methods enable the identification of signals, but do not allow for any conclusion on a causal link, for which a medical and pharmacological evaluation remains essential. Moreover, a real-time medical and pharmacological analysis is crucial in this type of health crisis.

\section{Declarations}

Funding No sources of funding was received for the preparation of this article.

Conflicts of interest Aurélie Grandvuillemin, Milou-Daniel Drici, Annie Pierre Jonville-Bera, Joelle Micallef, Jean Louis Montastruc, and the French Pharmacovigilance Network have no conflicts of interest that are directly relevant to the content of this article.

Ethics approval Not applicable.

Consent to participate Not applicable.

Consent for publication Not applicable.

Availability of data and materials Not applicable.

Code availability Not applicable.

Author contributions AG wrote the manuscript. MDD, APJB, JM, and JLM reviewed the manuscript. All authors read and approved the final version.

\section{References}

1. Lechien JR, Chiesa-Estomba CM, Place S, et al. Clinical and epidemiological characteristics of 1,420 European patients with mild-to-moderate coronavirus disease 2019. J Intern Med. 2020;288(3):335-44.

2. World Health Organization. Coronavirus disease (COVID-19). Technical guidance. 2020. https://www.who.int/fr/emergencie s/diseases/novel-coronavirus-2019/technical-guidance/namin g-the-coronavirus-disease-(covid-2019)-and-the-virus-thatcauses-it. Accessed 30 Nov 2020.

3. Haut Conseil de la Santé Publique. Avis relatif à la prise en charge des cas confirmés d'infection au virus SARS-CoV2. 5. 2020. https://www.hcsp.fr/Explore.cgi/avisrapportsdom aine?clefr $=771$. Accessed 30 Nov 2020.

4. WHO R\&D Blueprint. Informal consultation on prioritization of candidate therapeutic agents for use in novel coronavirus 2019 infection. 2020. https://apps.who.int/iris/bitstream/handl e/10665/330680/WHO-HEO-RDBlueprint\%28nCo V\%292020.1-eng.pdf?ua=1. Accessed 30 Nov 2020. 
5. de Wit E, van Doremalen N, Falzarano D, Munster VJ. SARS and MERS: recent insights into emerging coronaviruses. Nat Rev Microbiol. 2016;14(8):523-34.

6. Gupta A, Madhavan MV, Sehgal K, et al. Extrapulmonary manifestations of COVID-19. Nat Med. 2020;26(7):1017-32.

7. Lapeyre-Mestre M, Boucher A, Daveluy A, Gibaja V, Jouanjus E, Mallaret M, et al. French Addictovigilance Network. Addictovigilance contribution during COVID-19 epidemic and lockdown in France. Therapie. 2020;75(4):343-54.

8. Lane JCE, Weaver J, Kostka K, Duarte-Salles T, Abrahao MTF, Alghoul $\mathrm{H}$, et al. Risk of hydroxychloroquine alone and in combination with azithromycin in the treatment of rheumatoid arthritis: a multinational, retrospective study. Lancet Rheumatol. 2020;2(11):e698-711.

9. Gérard A, Romani S, Fresse A, Viard D, Parassol N, Granvuillemin A, et al. French Network of Pharmacovigilance Centers. "Off-label" use of hydroxychloroquine, azithromycin, lopinavirritonavir and chloroquine in COVID-19: a survey of cardiac adverse drug reactions by the French Network of Pharmacovigilance Centers. Therapie. 2020;75(4):371-9.

10. ANSM. Plaquenil et kaletra: les traitements testés pour soigner les patients COVID-19 ne doivent être utilisés qu'à l'hôpital: point d'information. 2020. https://www.ansm.sante.fr/S-informer/Point
s-d-information-Points-d-information/Plaquenil-et-Kaletra-lestraitements-testes-pour-soigner-les-patients-COVID-19-ne-doive nt-etre-utilises-qu-a-l-hopital-Point-d-information. Accessed 30 Nov 2020.

11. Haut Conseil de la Santé Publique. Avis relatif à l'utilisation de l'hydroxychloroquine dans le COVID-19. 2020. https://www.hcsp. fr/Explore.cgi/avisrapportsdomaine?clefr=837. Accessed $30 \mathrm{Nov}$ 2020.

12. US Food and Drug Administration. Press announcements. Coronavirus (COVID-19) update: FDA revokes emergency use authorization for chloroquine and hydroxychloroquine. https://www.fda. gov/news-events/press-announcements/coronavirus-covid-19update-fda-revokes-emergency-use-authorization-chloroquin e-and. Accessed 30 Nov 2020.

13. Micallef J, Soeiro T, Jonville-Béra AP. French Society of Pharmacology, Therapeutics (SFPT). Non-steroidal anti-inflammatory drugs, pharmacology, and COVID-19 infection. Therapie. 2020;75(4):355-62.

14. Micallef J, Soeiro T, Jonvillle-Béra AP. COVID-19 and NSAIDs: primum non nocere. Therapie. 2020;75(5):514-5. 